\title{
An Unprecedented 2-Dimensional Discrete-Time Fractional-Order System and Its Hidden Chaotic Attractors
}

\author{
Amina Aicha Khennaoui $\mathbb{D}^{1},{ }^{1}$ A. Othman Almatroud, ${ }^{2}$ Adel Ouannas, ${ }^{1}$ \\ M. Mossa Al-sawalha, ${ }^{2}$ Giuseppe Grassi, ${ }^{3}$ Viet-Thanh Pham, ${ }^{4}$ and Iqbal M. Batiha ${ }^{5}$ \\ ${ }^{1}$ Department of Mathematics and Computer Science, University of Larbi Ben M'hidi, Oum El Bouaghi, Algeria \\ ${ }^{2}$ Department of Mathematics, Faculty of Science, University of Ha'il, Ha'il 81451, Saudi Arabia \\ ${ }^{3}$ Universita Del Salento, Dipartimento Ingegneria Innovazione, Lecce 73100, Italy \\ ${ }^{4}$ Nonlinear Systems and Applications, Faculty of Electrical and Electronics Engineering, Ton Duc Thang University, \\ Ho Chi Minh City, Vietnam \\ ${ }^{5}$ Department of Mathematics, University of Jordan, Amman, Jordan
}

Correspondence should be addressed to Amina Aicha Khennaoui; kamina_aicha@yahoo.fr

Received 12 August 2020; Revised 30 October 2020; Accepted 22 December 2020; Published 6 January 2021

Academic Editor: Abdelalim Elsadany

Copyright (C) 2021 Amina Aicha Khennaoui et al. This is an open access article distributed under the Creative Commons Attribution License, which permits unrestricted use, distribution, and reproduction in any medium, provided the original work is properly cited.

\begin{abstract}
Some endeavors have been recently dedicated to explore the dynamic properties of the fractional-order discrete-time chaotic systems. To date, attention has been mainly focused on fractional-order discrete-time systems with "self-excited attractors." This paper makes a contribution to the topic of fractional-order discrete-time systems with "hidden attractors" by presenting a new 2 dimensional discrete-time system without equilibrium points. The conceived system possesses an interesting property not explored in the literature so far, i.e., it is characterized, for various fractional-order values, by the coexistence of various kinds of chaotic attractors. Bifurcation diagrams, computation of the largest Lyapunov exponents, phase plots, and the 0-1 test method are reported, with the aim to analyze the dynamics of the system, as well as to highlight the coexistence of chaotic attractors. Finally, an entropy algorithm is used to measure the complexity of the proposed system.
\end{abstract}

\section{Introduction}

Exploring chaotic dynamics has received considerable attention during the past few years [1]. Numerous attempts have been dedicated to analyze the classical systems (outlined by differential or difference equations of integer order), as well as fractional-order systems (outlined by differential or difference equations of fractional order) [2]. Generally speaking, regardless of the type of system, chaos can appear in the form of "hidden attractors" or "self-excited attractors" [3-6]. On the first occasion, the initial conditions, for the purpose of getting chaos, are situated near the saddle points of the motion [3], whereas, on the last occasion, the initial conditions may only be set up via a wide range of computerbased search [4], given that the corresponding dynamic systems are distinguished by the presence of stable equilibrium points [5] or else by the absence of them at all [6].

Referring to fractional-order chaotic discrete-time systems (i.e., systems outlined by difference equations of fractional order), many scholars have mainly focused on the system's dynamics characterized by the presence of "selfexcited attractors" [7, 8]. For example, the so-called generalized Hénon map of three dimensions has been studied in [9], while some dynamics of the fractionalized logistic map were examined in [10]. In [11], three different fractional-order discrete-time systems (FoDs) have been investigated, i.e., Wang's, Rossler's, and Stefanski's maps. In [12], the chaotic behaviors of the fractional-order sine and standard maps were analyzed, whereas in [13], the dynamic properties of the fractional-order Grassi-Miller map have been illustrated in 
detail. Additionally, the presence of chaos in the fractionalorder discrete double scroll map has been investigated in [14], whereas in [12], the fractional-order delayed logistic map was analyzed regarding to its chaotic behavior. It is worthy to state that all these FoDs have shown "self-excited attractors." On the other hand, very few FoDs characterized by "hidden attractors" have been investigated in the previously published works up to this time [15-19]. For example, in [15], the dynamics of the fractional-order version of the standard iterated map have been investigated, whereas in [18], a 2Dimensional FoDs (2D-FoDs) without discontinuity for all equations of the system has been presented. However, these FoMs with "hidden attractors" do not show any coexisting chaotic attractors. Based on these considerations, this paper aims to make a contribution to the topic of FoDs with "hidden attractors" by presenting a new 2D-FoD without equilibrium points. The conceived system possesses an interesting property, i.e., it is characterized by the coexistence of various kinds of chaotic attractors. Here is how this paper is arranged. Section 2 introduces a new 2D-FoD time system without equilibria, along with some primary preliminaries associated with discrete-time noninteger-order calculus. In Section 3, the dynamic properties of the conceived map are analyzed via bifurcation diagrams and computation of the Largest Lyapunov Exponents (LLEs). In Section 4, a $0-1$ test is reported to highlight the existence of chaotic hidden attractors. Also, an entropy algorithm is used to measure the complexity of the proposed system. Finally, a number of phase plots are reported, which highlight the coexistence of several types of chaotic attractors for various fractional-order values of the conceived system.

\section{A New 2D-FoDs}

This paper considers the following 2D-difference system:

$$
\left\{{ }^{c} \Delta_{a}^{\gamma} x(t)=y(t-1+\gamma)-x(t-1+\gamma),{ }^{c} \Delta_{a}^{\gamma}=-\alpha y(t-1+\gamma)-0.37 y^{2}(t-1+\gamma)+0.81 x(t-1+\gamma) y(t-1+\gamma)+1.79\right.
$$

where $x$ and $y$ stand for state variables of the FoDs, $\alpha$ is the system's parameter, and ${ }^{c} \Delta_{a}^{\gamma}$ is the Caputo-like difference operator of fractional-order $\gamma$, where $\gamma \in] 0,1]$.

Next, two main definitions that will pave the way for obtaining novel results are given below for completeness. Such two definitions are stated for the ${ }^{c} \Delta_{a}^{\gamma}$ in its $\gamma^{\text {th }}$ - order version and also for the $\gamma^{\text {th }}$-fractional sum operator, $\Delta^{-\gamma}$, respectively.

Definition 1. Let $\gamma>0$ and $\mathbf{y}(\mathbf{t}) \in \mathbb{N}_{a}$. We define the $\gamma^{\text {th }}$-order Caputo-like operator as [20]

$$
{ }^{c} \Delta_{a}^{\gamma} y(t)=\frac{1}{\Gamma(1-\gamma)} \sum_{\tau=a}^{t-(1-\gamma)}(t-\tau-1)^{-\gamma} \Delta_{\tau} y(\tau),
$$

where $\Gamma($.$) denotes the gamma function and t \in \mathbb{N}_{a+1-\gamma}$.

Definition 2. Let $\gamma>0$; we define the $\gamma^{\text {th }}$-fractional sum, $\Delta^{-\gamma}$ as

$$
\Delta_{a}^{-\gamma} y(t)=\frac{1}{\Gamma(\gamma)} \sum_{\tau=a}^{t-\gamma}(t-\tau-1)^{(\gamma-1)} \Delta_{\tau} y(\tau) .
$$

Using $\Delta^{-\gamma}$ makes (1) to be also rewritten as an integral equation in the Volterra sense as follows:

$$
\left\{\begin{array}{l}
x(t)=x_{0}+\frac{1}{\Gamma(\gamma)} \sum_{\tau=a+1-\gamma}^{t-\gamma}(t-\tau-1)^{(\gamma-1)}(y(\tau+\gamma-1)-x(\tau+\gamma-1)), \\
y(t)=y_{0}+\frac{1}{\Gamma(\gamma)} \sum_{\tau=a+1-\gamma}^{t-\gamma}(t-\tau-1)^{(\gamma-1)}\left(\begin{array}{c}
-\alpha y(\tau+\gamma-1)-0.37 y^{2}(\tau+\gamma-1)+ \\
0.81 x(\tau+\gamma-1) y(\tau+\gamma-1)+1.79
\end{array}\right) .
\end{array}\right.
$$

In the present work, some numerical methods are adopted to examine the complex dynamics of the proposed FoDs. First of all, we discuss the equilibrium points of the model at hand. Actually, the equilibrium points can be determined by finding the solution of the following system:

$$
\left\{\begin{array}{l}
y-x=0 \\
-\alpha y-0.37 y^{2}+0.81 x y+1.79=0 .
\end{array}\right.
$$

From system (5), it follows that

$$
-\alpha y-1.18 y^{2}+1.79=0
$$


Thus, FoDs (1) has no equilibrium point when $-2.9067<\alpha<2.9067$. This result shows that FoDs (1) is able to produce a chaotic hidden attractor for appropriate choice of initial conditions and fractional order as well.
Secondly, we present the numerical formulae corresponding to all equations given in FoDs (1). This is can be carried out by first setting the initial point $a$ to be equal to 0 , then assuming $\tau+\gamma=\kappa$, and finally, replacing $(t-\tau-1)^{(\gamma-1)} / \Gamma(\gamma)$ by $\Gamma(t-\tau) /(\Gamma(\gamma) \Gamma(t-\tau-\gamma+1))$. Thus, (4) becomes

$$
\left\{\begin{array}{l}
x(n)=x_{0}+\frac{1}{\Gamma(\gamma)} \sum_{\kappa=1}^{n} \frac{\Gamma(n-\kappa+\gamma)}{\Gamma(n-\kappa+1)}(y(\kappa-1)-x(\kappa-1)), \\
y(n)=y_{0}+\frac{1}{\Gamma(\gamma)} \sum_{\kappa=1}^{n} \frac{\Gamma(n-\kappa+\gamma)}{\Gamma(n-\kappa+1)}\left(-\alpha y(\kappa-1)-0.37 y^{2}(\kappa-1)+0.81 x(\kappa-1) y(\kappa-1)+1.79\right),
\end{array}\right.
$$

where $x_{0}$ and $y_{0}$ are the initial states. According to the discrete equation (7), the proposed fractional system (1) has memory effects, which means that the iterated solutions $x$ and $y$ are determined by all the previous states. In the next section, some dynamic characteristics of the novel $2 \mathrm{D}-\mathrm{FoD}$ system are analyzed numerically.

\section{Bifurcations and LLEs}

When plotting bifurcation diagrams, two sets of symmetrical initial states are considered. The bifurcation diagram is plotted in blue for the initial state $x_{0}=1.78, y_{0}=-0.79$ and in red for the initial states $x_{0}=-1.78, y_{0}=0.79$.

\subsection{Bifurcation and LLEs versus the System's Parameter $\alpha$.} Firstly, the bifurcation diagram of FoDs (1) is studied as $\alpha$ varies from 1.35109 to 1.9199 . Besides, the bifurcation diagrams and LLEs of the state variable $x(n)$ are also studied corresponding to two distinct fractional-order values of $\gamma$, as exhibited in Figures 1 and 2. It can be seen that the states of FoDs (1) change qualitatively with the variation of $\alpha$ and $\gamma$. In particular, the bifurcation diagram of FoDs (1) is illustrated in Figure 1(a), for $\gamma=0.9362$. When $\alpha$ increases from 1.35109 to 1.9199 , the states of the system go, via period-doubling bifurcation, to chaotic motion. It is noteworthy that FoDs (1) exhibits chaotic behavior in larger intervals for the initial condition $x_{0}=1.78, y_{0}=-0.79$. As shown in Figure 2, when $\gamma$ is increased starting from 0.9362 up to 0.992 , FoDs (1) shows chaotic motion over most of the range $(1.7387,1.9136)$.

3.2. Bifurcation versus Fractional-Order $\gamma$. In order to highlight the effect of $\gamma$ on the dynamic behavior of FoDs (1), its bifurcation with respect to $\gamma$ too is considered. We fix the parameter $\alpha$ to be equal to 1.73 and change $\gamma$ within $[0,1]$. The bifurcation diagram and the LLE are illustrated in Figures 3(a) and 3(b), respectively. As one can see, the system has positive LE when $\gamma$ takes the smallest values, indicating that FoDs (1) is chaotic. Besides, when $\gamma \in[0.9362,0.9402] \cup] 0.9816,0.9834]$, FoDs (1) shows chaotic behavior. The phase diagrams are plotted in Figure 4 for different values of $\gamma$. From these diagrams, it is clear that as the value of $\gamma$ increases, different chaotic attractors are observed. Moreover, these figures indicate that the fractional order $\gamma$ is another bifurcation parameter.

3.3. Coexisting Chaotic Attractors. Herein, the dynamics of FoDs (1) are analyzed using the phase portraits, obtained by fixing the parameter $\alpha$ and by considering the two previous different sets of initial conditions. For $\gamma=0.992$, as shown in Figure 5(a), FoDs (1) highlights the coexistence of a hidden chaotic attractor corresponding to the two initial conditions $(1.78,-0.79)$. Similarly, when the order $\gamma$ is selected to be equal 0.9992 in FoDs (1), Figure 5(b) highlights the coexistence chaotic hidden attractors corresponding to the two initial conditions $(-1.78,0.79)$ and $(1.78,-0.79)$, respectively. Finally, when $\gamma$ is taken to be equal to 0.96 , the coexisting chaotic hidden attractors are plotted as in Figure 5(c). One might deduce that the dynamic behavior of the new FoDs given in (1) is complex and interesting, by virtue of the presence of different types of coexisting hidden chaotic attractors.

\section{Test for Chaos and Approximate Entropy}

In the following section, we present the influence of both fractional-order and initial-conditions on the dynamical behavior of the suggested discrete-time system by considering the 0-1 test method. Then, we introduce the approximate entropy to further investigate the complexity of fractional-order discrete-time system (1).

4.1. Test for Chaos. To reflect the sensitivity of the FoDs, the 0-1 test is considered. This test was proposed in [21] for fractionalorder systems to distinguish regular and chaotic dynamics. As opposed to the Lyapunov exponents method, the 0-1 test is applied to known or unknown systems regarding the phase plane. Thus, it is able to identify the chaos in a series of data where the phase space reconstruction is not necessary. For model (1), this method works for the finite points $\left(y_{i}\right)_{i=1, \ldots, N}$ and is a suitable choice of $c \in(0,2 \pi)$. Using the approach in [21], one can define the two terms for $m=\overline{1, N}$ as 


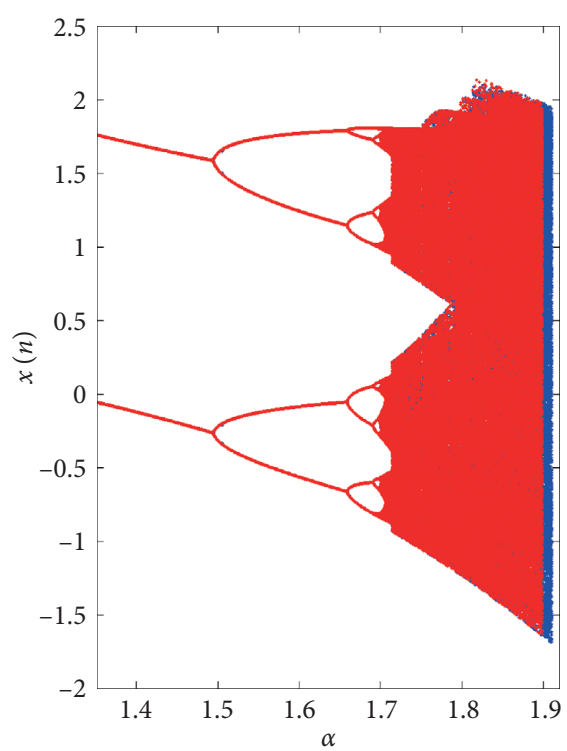

(a)

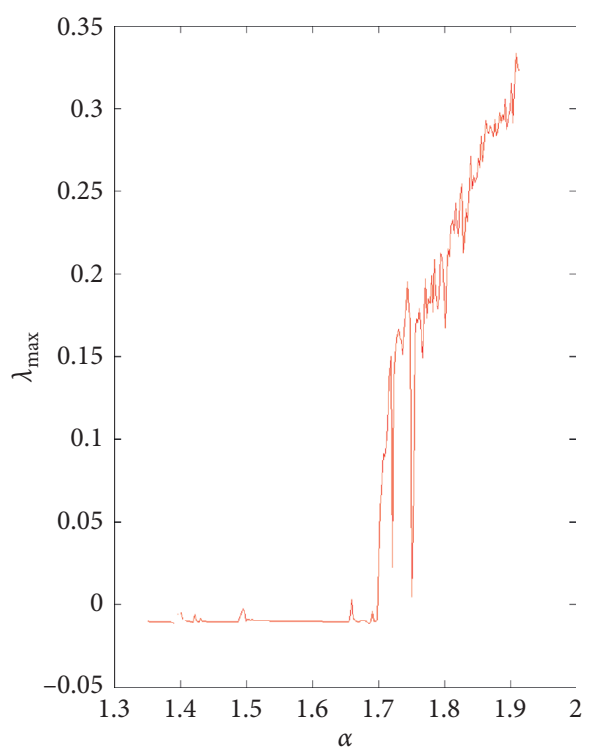

(b)

Figure 1: (a) Bifurcation diagrams of FoDs (1) vs. $\alpha$ when $\gamma=0.9362$; (b) LLE diagram according to (a).

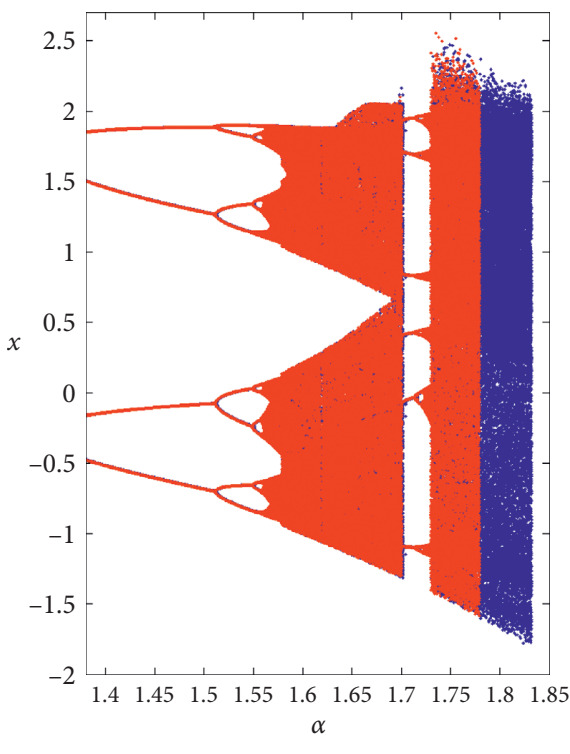

(a)

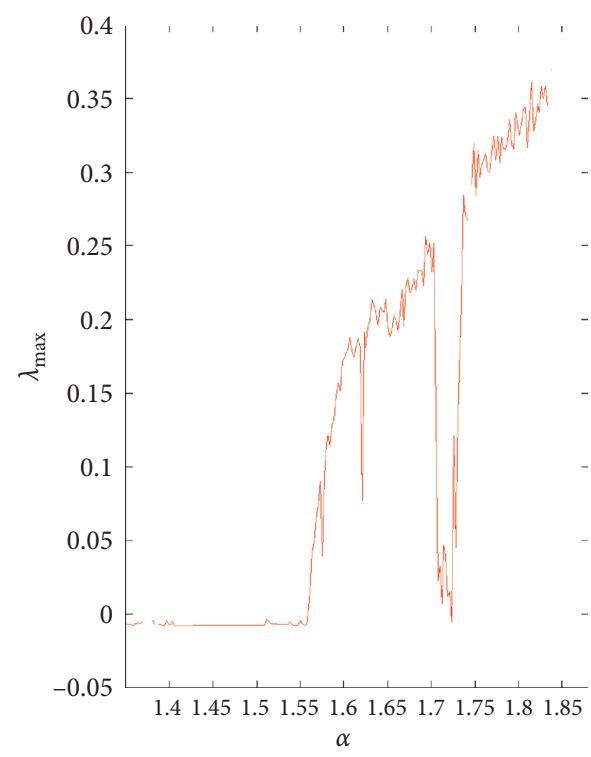

(b)

Figure 2: (a) Bifurcation diagrams of FoDs (1) vs. $\alpha$ when $\gamma=0.992$; (b) LLE diagram according to (a).

$$
\begin{aligned}
& p_{m}=\sum_{i=1}^{m} y_{i} \cos (i c), \\
& s_{m}=\sum_{i=1}^{m} y_{i} \sin (i c) .
\end{aligned}
$$

Such terms are called the translation components. In order to study the boundedness or unboundedness of the functions $p_{m}$ and $s_{m}$, we calculate the time-averaged meansquare displacement, which can be defined as

$$
M_{m}=\lim _{N \longrightarrow+\infty} \frac{1}{N} \sum_{j=1}^{N}\left(\left(p_{j+m}-p_{j}\right)^{2}+\left(s_{j+m}-s_{j}\right)^{2}\right) .
$$

In practice $n \ll N$. Finally, we obtain the asymptotic growth rate $K$ via

$$
K=\operatorname{median}\left(K_{c}\right),
$$

where $K_{c}=\lim _{m \rightarrow \infty}\left(\log M_{m} / \log m\right)$.

On the other hand, the " $0-1$ test" has been developed in [22], such that the output $K$ of the test is obtained using 


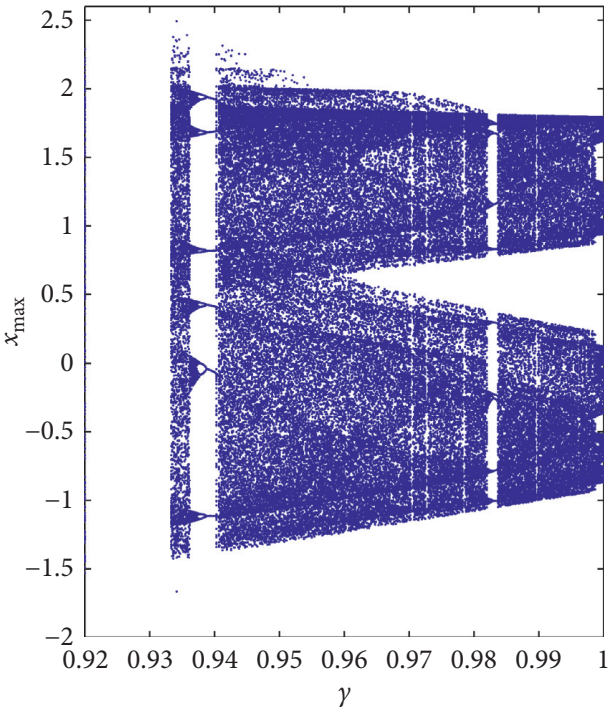

(a)

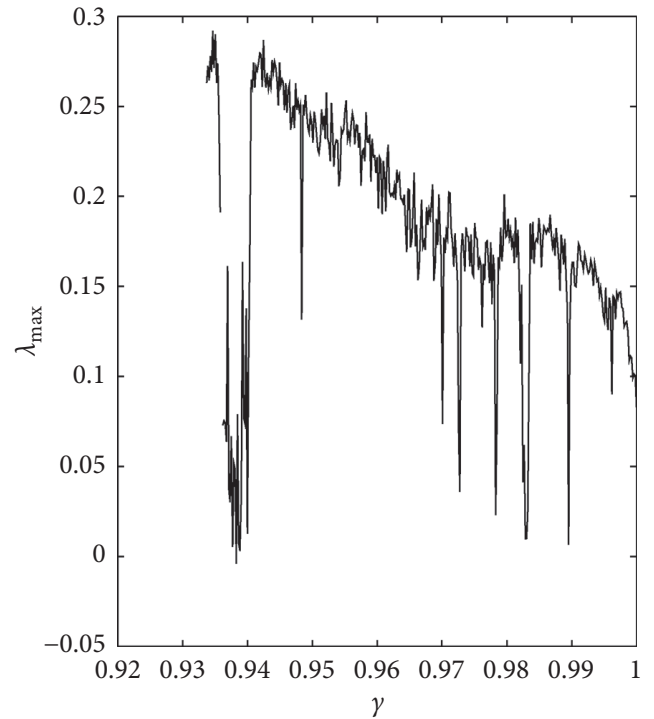

(b)

Figure 3: (a) Bifurcation diagram vs. $\gamma$, when $\alpha=1.73$; (b) LLE according to diagram (a).
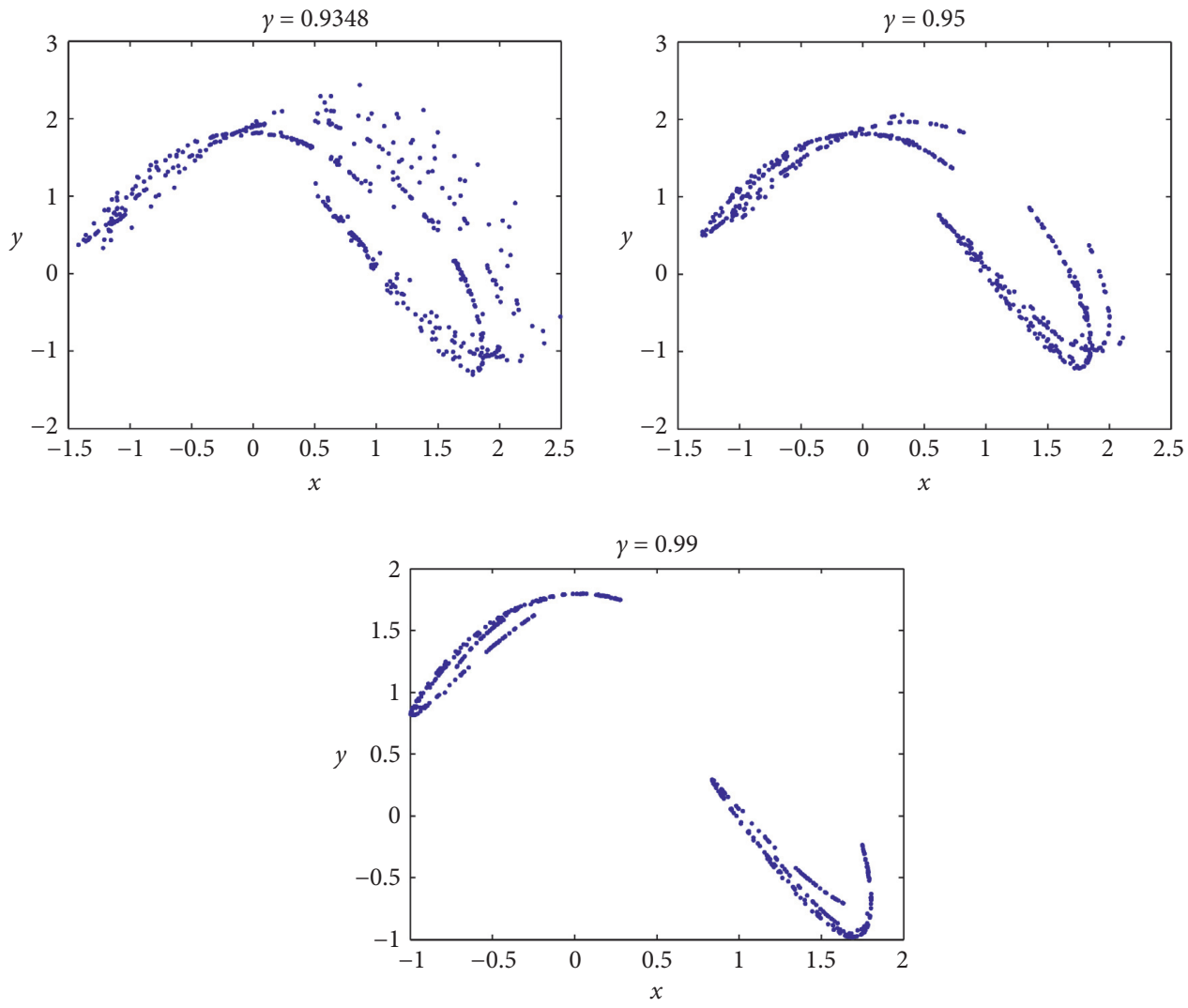

Figure 4: Phase diagrams of fractional-order map (1) for different values of fractional order.

correlation to measure the growth rate of the mean-square displacement $D_{m}$ for better convergence property. Generally, $D_{m}$ is calculated as

$$
D_{m}=M_{m}-V_{\text {osc }} \text {, }
$$

where $V_{\text {osc }}$ is the oscillatory term:

$$
V_{\text {osc }}(c, n)=\left(\lim _{N \longrightarrow \infty} \frac{1}{N} \sum_{j=1}^{N} x(j)\right)^{2} \frac{1-\cos (m c)}{1-\cos (c)} .
$$




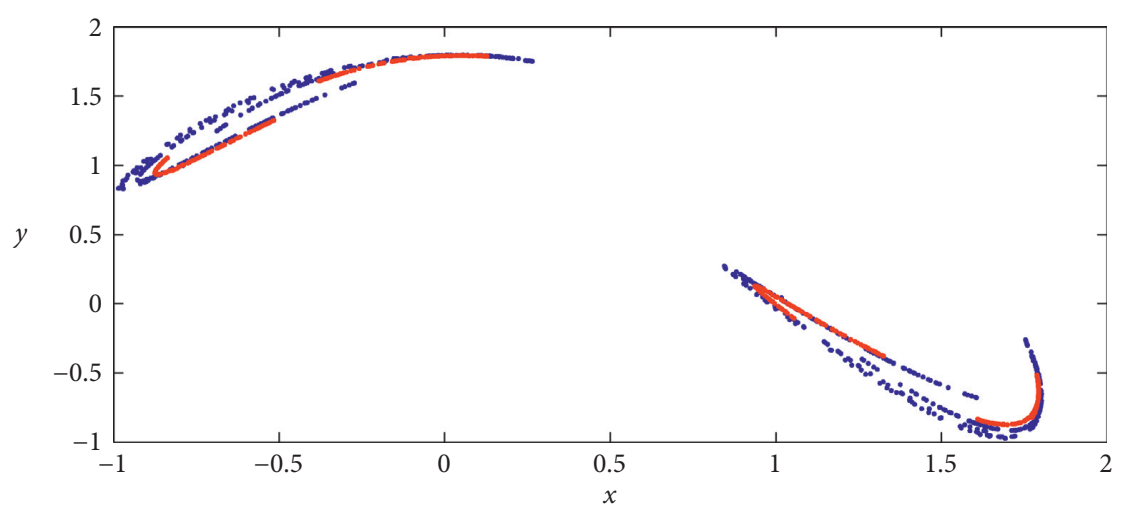

(a)

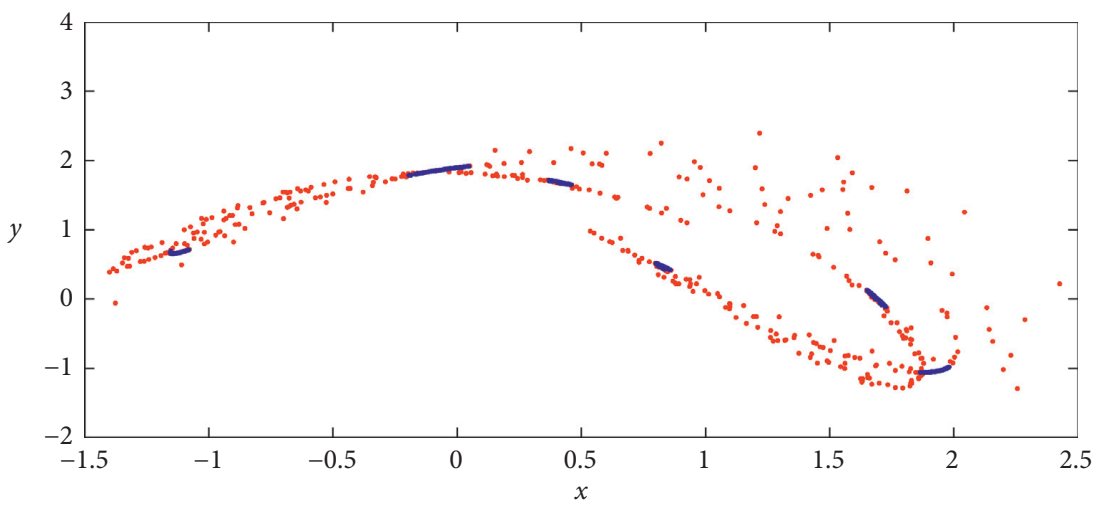

(b)

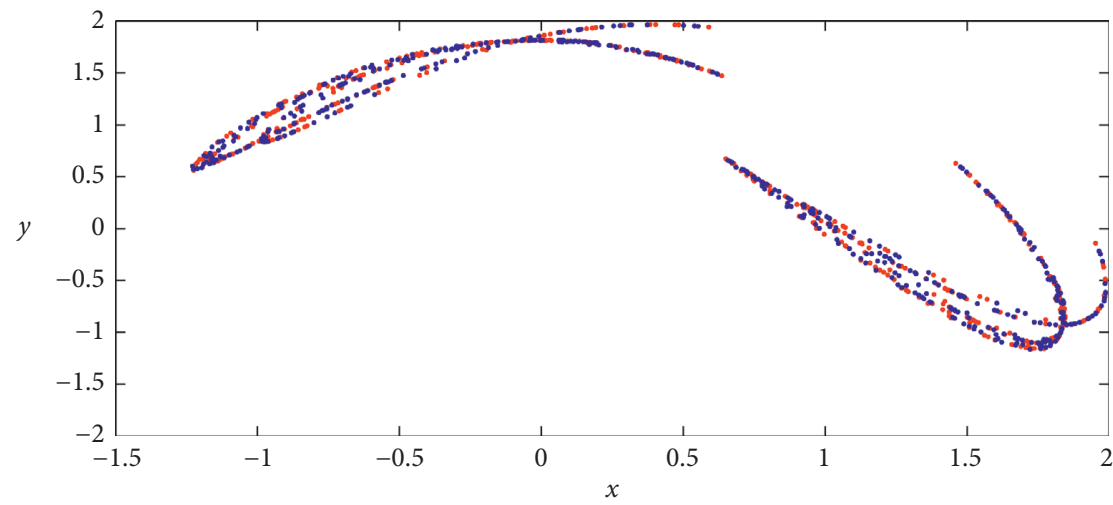

(c)

Figure 5: The coexisting attractors of FoDs (1) with $\alpha=1.73$ and subject to the two initial conditions $(-1.78,0.79)$ and $(1.78,-0.79)$ for the red and the blue attractors, respectively: (a) $\gamma=0.9992$; (b) $\gamma=0.9362$; and (c) $\gamma=0.96$.

It is shown in [22] that the modified mean-square deplacement $D_{c}$ processes better convergence than $M_{c}$. Therefore, the output $K$ can now be performed as the covariance $\operatorname{cov}(x, y)=(1 / m) \sum_{i=1}^{m}(x(i)-\bar{x})(y(i)-\bar{y})$ and variation $\operatorname{var}(x)=\operatorname{cov}(x, x)$ of $m$ element as follows:

$$
K_{c}=\frac{\operatorname{cov}(r, s)}{\sqrt{\operatorname{var}(r) \operatorname{var}(s)}} \in[1,1],
$$

where $r=\{1,2, \ldots, m\}$ and $s$ is the vector formed by the mean-square displacement $D_{m}$.
In both methods, fractional-order discrete-time system (1) is evaluated to be chaotic if the plot of $p$ and $s$ in the $p-s$ plane present Brownian-like trajectories and if $K$ approaches 1 , while it becomes regular as $K$ approaches 0 , and $p$ and $s$ display bounded-like trajectories. Figure 6, however, depicts the results of the test for different values of fractional order $\gamma$ in which $\left(x_{0}, y_{0}\right)=(-1.78,0.79)$. Based on this figure, one can observe that the output $K$ has appeared in a similar manner to the results of the maximum LE and bifurcation diagram, shown in Figure 3, which clearly confirms the abovementioned results. 


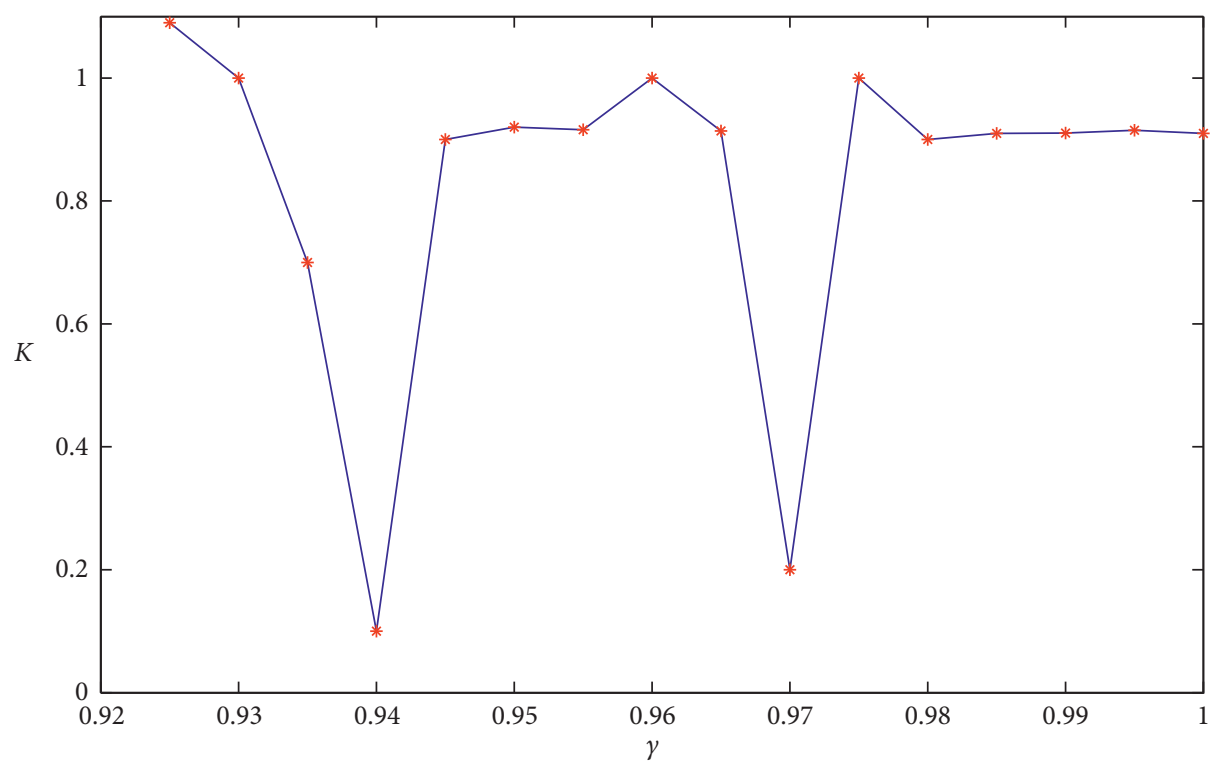

Figure 6: Asymptotic growth rate $K$ vs. $\gamma$, when $\alpha=1.73$.
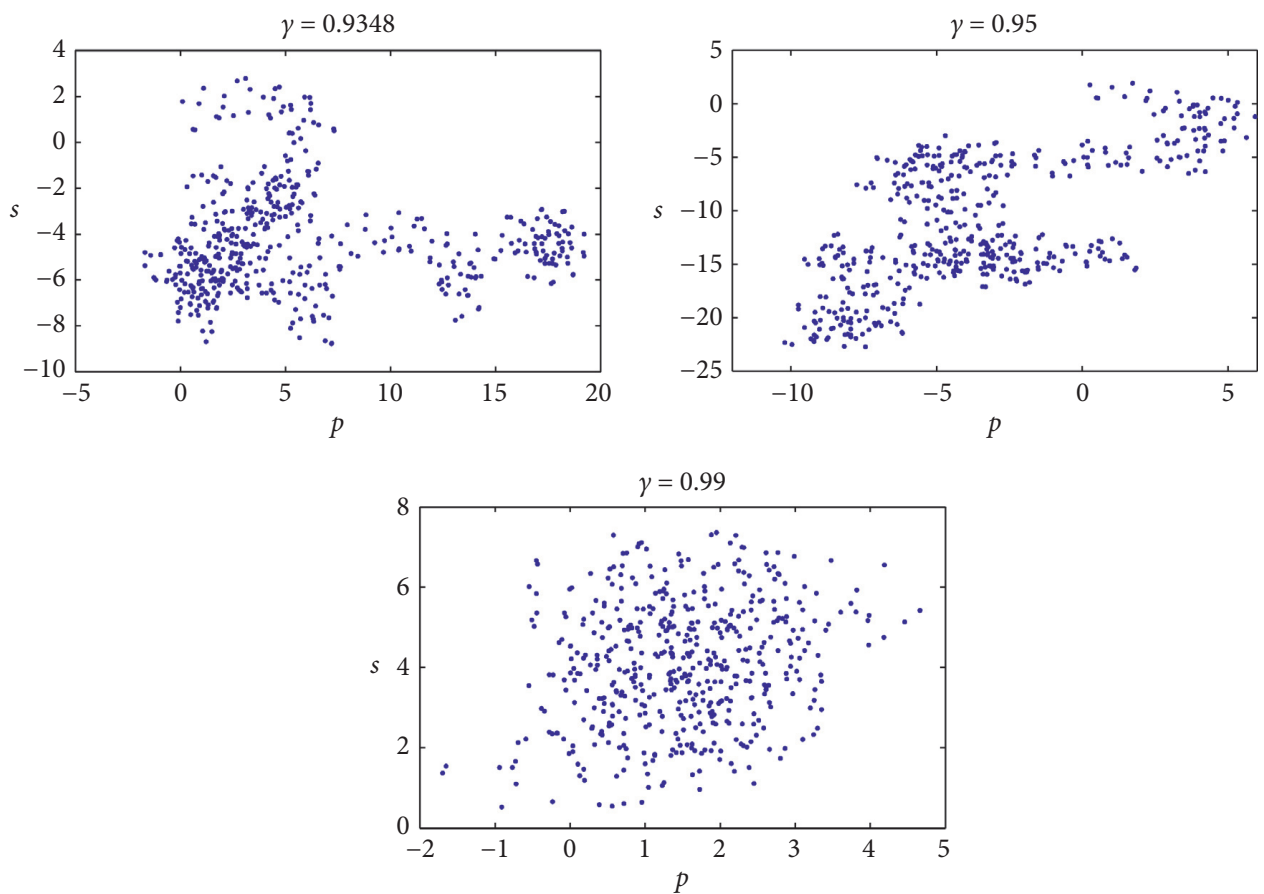

Figure 7: $0-1$ test of the FoDs $(1)$ for $\alpha=1.73$ and subject to the initial conditions $(-1.78,0.79)$ and for different values of $\gamma$.

Next, the translations functions $p$ and $s$ of the 0 - 1 test for different fractional-order values are plotted in Figure 7, and it fits well with the phase diagrams in Figure 4. In particular, Figure 7 depicts the Brownian-like trajectories for all the three fractionalorder values indicating that the suggested map is chaotic in this case. To further confirm the results, we choose to plot a $3 \mathrm{D}$ view of the asymptotic growth rate $K$ of the $0-1$ test when $1.3<\alpha \leq 1.9$ and by varying $\gamma$ from 0.92 to 1 (see Figure 8 ). It is clear that the dynamics of system (1) shift to small intervals of $\alpha$ as the fractional order $\gamma$ decreases and disappears as the fractional order and system parameter $\alpha$ values decrease.
4.2. Approximate Entropy. The approximate entropy (ApEn) [23] is the measurement of the degree of complexity of a series of data from a multidimensional perspective. This method estimates the regularity by assigning a nonnegative number, where higher values indicate higher complexity. By applying the technique in [23], we consider $\left(x_{i}\right)_{i=1, . ., N}$ points that are obtained from discrete formula (4). The value of the approximate entropy depends on two important parameters, i.e., $m$ and $\tau$, where the input $\tau$ is the similar tolerance whereas $m$ is the embedding dimension. We reconstruct a subsequence of $x$ such that $\chi(i)=[x(i), \ldots, x(i+m-1)]$, where 


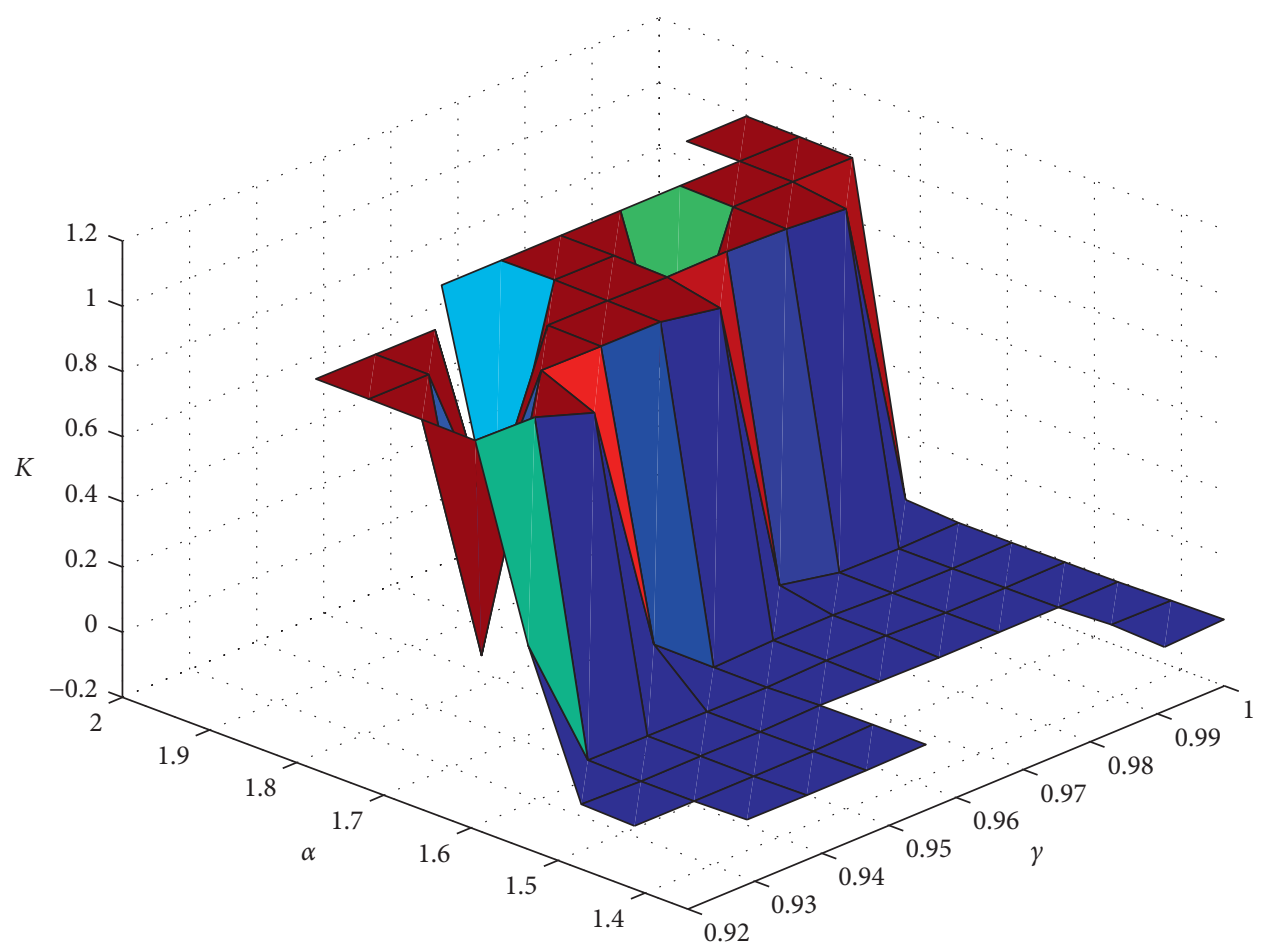

Figure 8: Asymptotic growth rate of the 0-1 test method of the fractional-order map (1) in three-dimensional space with the variation of system parameter $\alpha$ and fractional order $\gamma$.

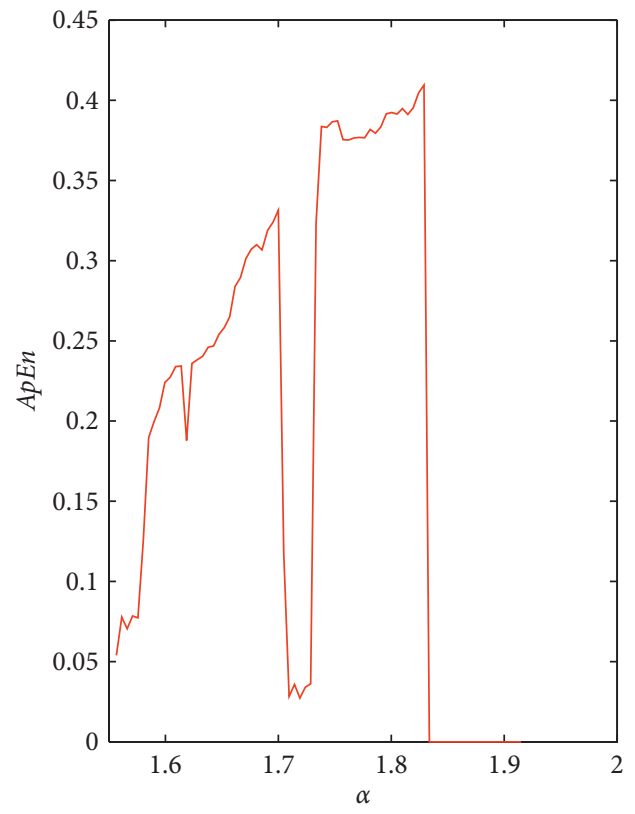

(a)

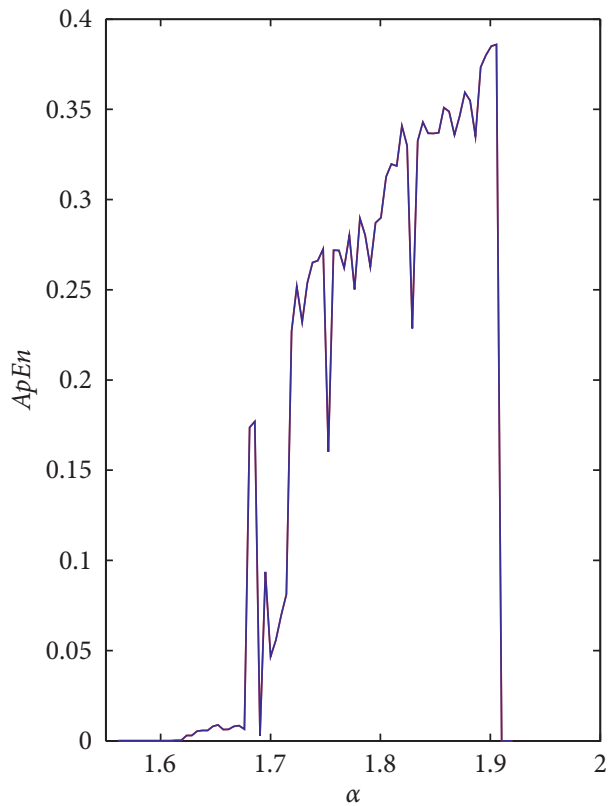

(b)

Figure 9: The approximate entropy (ApEn) of FoDs (1) versus $\alpha$ for (a) $\$ \gamma=0.9362$ and (b) $\gamma=0.992$.

$m$ presents the points from $x(i)$ to $x(i+m-1)$. Let $K$ be the number of $\chi(i)$ such that the maximum absolute difference of two vectors $\chi(i)$ and $\chi(j)$ is lower or equal to the tolerance $\tau$.
The relative frequency of $\chi(i)$ is similar to $\chi(j)$, and it has the form $C_{i}^{m}(\tau)=K /(N-m+1)$. From $C_{i}^{m}$, we calculate the logarithm and then define the average for all $i$ as follows: 


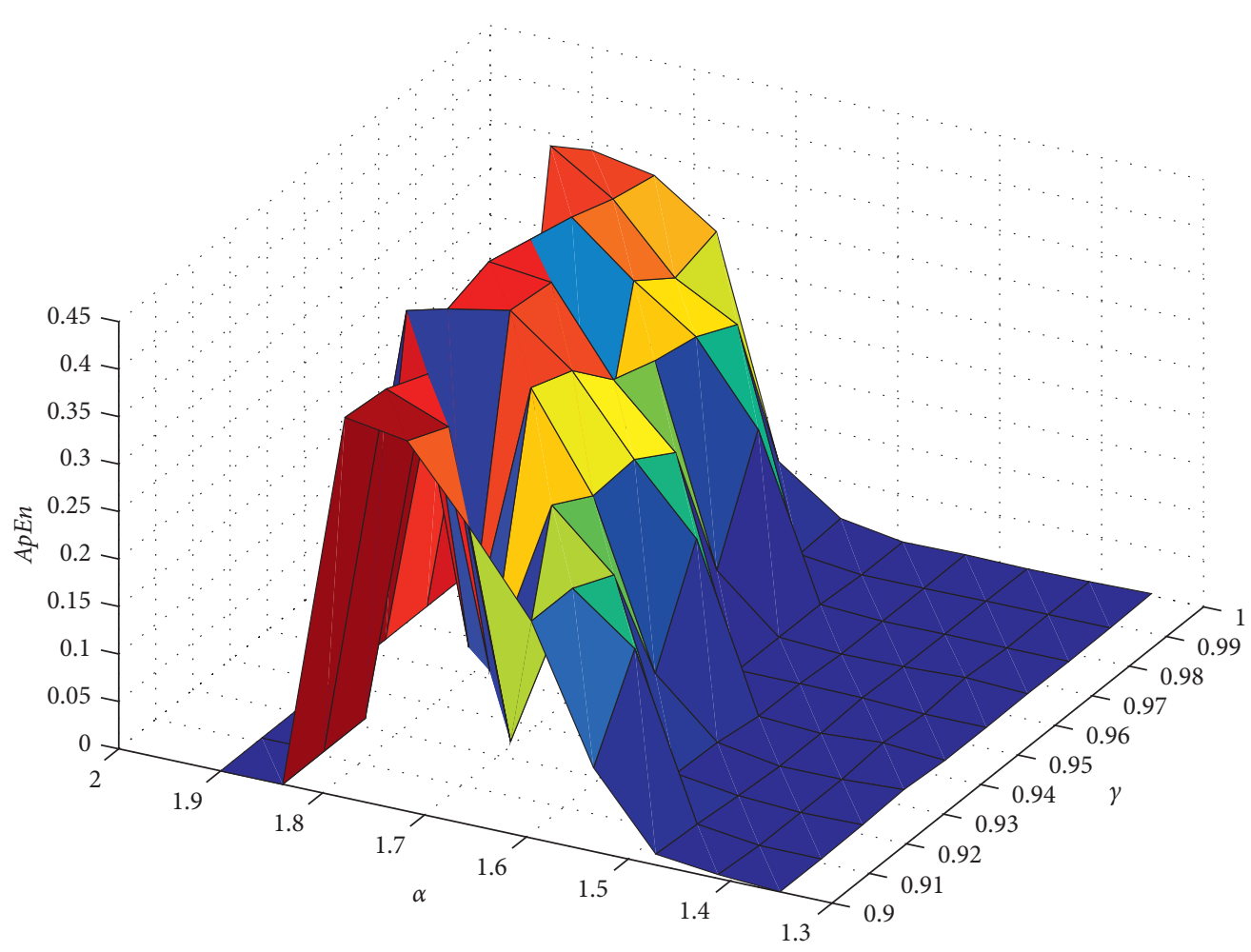

Figure 10: The approximate entropy (ApEn) of the fractional-order map (1) in three-dimensional space with the variation of system parameter $\alpha$ and fractional order $\gamma$.

$$
\phi^{m}(r)=\frac{1}{N-m-1} \sum_{i=1}^{N-m+1} \log C_{i}^{m}(r) .
$$

Thus, the approximate entropy of order $m$ is set as

$$
\operatorname{ApEn}=\phi^{m}(r)-\phi^{m+1}(r) .
$$

Herein, the structural complexity of FoDs (1) is analysed via equation (15) by varying the control parameter $\alpha$ and the fractional order $\gamma$ as reported in Figures 9 and 10. In particular, the approximate entropy (ApEn) diagrams with two different initial conditions are plotted in Figure 9. It can be seen that the complexity of FoDs (1) strongly depends on the variations of $\gamma$ and $\alpha$. In particular, Figure 10 highlights that there are some combined values of $\alpha$ and $\gamma$ for which the approximate entropy ApEn is high, indicating that FoDs (1) is characterized by complex dynamic behaviors for both initial conditions. The results agree will with the bifurcation diagrams in Figures 1 and 2.

\section{Conclusions}

Referring to a fractional-order discrete-time system (FoDs) with "hidden attractors," this paper has introduced a new 2D system without equilibrium points. The system possesses the interesting property of being characterized by the coexistence of various kinds of chaotic attractors, for various fractional-order values. Bifurcation diagrams, computation of the Largest Lyapunov Exponents (LLEs), and phase plots have been reported to investigate the dynamics of the map, indicating the effectiveness of the approach developed herein along with the 0-1 test. Finally, an entropy algorithm is used to measure the complexity of the proposed system.

\section{Data Availability}

The data that support the findings of this study are available within the article.

\section{Conflicts of Interest}

The authors declare no conflicts of interest.

\section{Authors' Contributions}

Adel Ouannas, Amina-Aicha Khennaoui, A. Othman Almatroud, and Iqbal M. Batiha conceptualized the study; data curation was performed by Amina-Aicha Khennaoui, M. Mossa Al-sawalha, Adel Ouannas, and Viet-Thanh Pham; Amina-Aicha Khennaoui, A. Othman Almatroud, Viet-Thanh Pham, and Iqbal M. Batiha conducted investigation; Adel Ouannas and Giuseppe Grassi formulated the methodology; Giuseppe Grassi supervised the work; and Adel Ouannas and Iqbal M. Batiha were involved in validation.

\section{Acknowledgments}

This work was supported by the Scientific Research Deanship at University of Ha'il- Saudi Arabia, though Project No. RG-191307. 


\section{References}

[1] J. C. Sprott, Elegant Chaos: Algebraically Simple Chaotic Flows, World Scientific, Singapore, 2010.

[2] G. M. Zaslavsky and G. M. Zaslavskij, Hamiltonian Chaos and Fractional Dynamics, Oxford University Press on Demand, Oxford, England, 2005.

[3] G. A. Leonov, N. V. Kuznetsov, and V. I. Vagaitsev, "Hidden attractor in smooth Chua systems," Physica D: Nonlinear Phenomena, vol. 241, no. 18, pp. 1482-1486, 2012.

[4] G. A. Leonov and N. V. Kuznetsov, "Hidden attractors in dynamical systems. From hidden oscillations in hilbertKolmogorov, Aizerman, and Kalman problems to hidden chaotic attractor in chua circuits," International Journal of Bifurcation and Chaos, vol. 23, no. 01, p. 1330002, 2013.

[5] M. Molaie, S. Jafari, J. C. Sprott, and S. M. R. H. Golpayegani, "Simple chaotic flows with one stable equilibrium," International Journal of Bifurcation and Chaos, vol. 23, no. 11, p. 1350188, 2013.

[6] Z. Wei, "Dynamical behaviors of a chaotic system with no equilibria," Physics Letters A, vol. 376, no. 2, pp. 102-108, 2011.

[7] A. Ouannas, A.-A. Khennaoui, Z. Odibat, V.-T. Pham, and G. Grassi, "On the dynamics, control and synchronization of fractional-order Ikeda map," Chaos, Solitons \& Fractals, vol. 123, pp. 108-115, 2019.

[8] A.-A. Khennaoui, A. Ouannas, S. Bendoukha, G. Grassi, R. P. Lozi, and V.-T. Pham, "On fractional-order discretetime systems: chaos, stabilization and synchronization," Chaos, Solitons \& Fractals, vol. 119, pp. 150-162, 2019.

[9] L. Jouini, A. Ouannas, A. A. Khennaoui, X. Wang, G. Grassi, and V. T. Pham, "The fractional form of a new three-dimensional generalized Henon map," Advances in Difference Equations, vol. 2019, no. 1, p. 122, 2019.

[10] G. C. Wu and D. Baleanu, "Discrete fractional logistic map and its chaos," Nonlinear Dynamics, vol. 75 , no. 1-2, pp. 283-287, 2014.

[11] A. A. Khennaoui, A. Ouannas, S. Bendoukha et al., "Chaos, control, and synchronization in some fractional-order difference equations," Advances in Difference Equations, vol. 2019, no. 1, pp. 1-23, 2019.

[12] G.-C. Wu and D. Baleanu, "Discrete chaos in fractional delayed logistic maps," Nonlinear Dynamics, vol. 80, no. 4, pp. 1697-1703, 2015.

[13] A. Ouannas, A.-A. Khennaoui, G. Grassi, and S. Bendoukha, "On chaos in the fractional-order Grassi-Miller map and its control," Journal of Computational and Applied Mathematics, vol. 358, pp. 293-305, 2019.

[14] A. Ouannas, A.-A. Khennaoui, S. Bendoukha, and G. Grassi, "On the dynamics and control of a fractional form of the discrete double scroll," International Journal of Bifurcation and Chaos, vol. 29, no. 6, Article ID 1950078, 2019.

[15] A. Ouannas, X. Wang, A.-A. Khennaoui, S. Bendoukha, V.-T. Pham, and F. Alsaadi, "Fractional form of a chaotic map without fixed points: chaos, entropy and control," Entropy, vol. 20, no. 10, p. 720, 2018.

[16] A. A. Khennaoui, A. Ouannas, S. Boulaaras, V.-T. Pham, and A. Taher Azar, "A fractional map with hidden attractors: chaos and control," The European Physical Journal Special Topics, vol. 229, no. 6-7, pp. 1083-1093, 2020.

[17] A. Ouannas, A. A. Khennaoui, S. Momani, G. Grassi, and V. T. Pham, "Chaos and control of a three-dimensional fractional order discrete-time system with no equilibrium and its synchronization," AIP Advances, vol. 10, no. 4, p. 045310, 2020.

[18] A. Ouannas, A.-A. Khennaoui, S. Momani et al., "A quadratic fractional map without equilibria: bifurcation, 0-1 test, complexity, entropy, and control," Electronics, vol. 9, no. 5, p. 748, 2020.

[19] A. Ouannas, A. A. Khennaoui, S. Momani, V. T. Pham, and R. El-Khazali, "Hidden attractors in a new fractional-order discrete system: chaos, complexity, entropy, and control," Chinese Physics B, vol. 29, no. 5, p. 050504, 2020.

[20] T. Abdeljawad, "On Riemann and Caputo fractional differences," Computers \& Mathematics with Applications, vol. 62, no. 3, pp. 1602-1611, 2011.

[21] D. Cafagna and G. Grassi, "An effective method for detecting chaos in fractional-order systems," International Journal of Bifurcation and Chaos, vol. 20, no. 3, pp. 669-678, 2010.

[22] G. A. Gottwald and I. Melbourne, "A new test for chaos in deterministic systems," Proceedings of the Royal Society of London. Series A: Mathematical, Physical and Engineering Sciences, vol. 460, no. 2042, pp. 603-611, 2004.

[23] S. M. Pincus, "Approximate entropy as a measure of system complexity," Proceedings of the National Academy of Sciences, vol. 88, no. 6, pp. 2297-2301, 1991. 